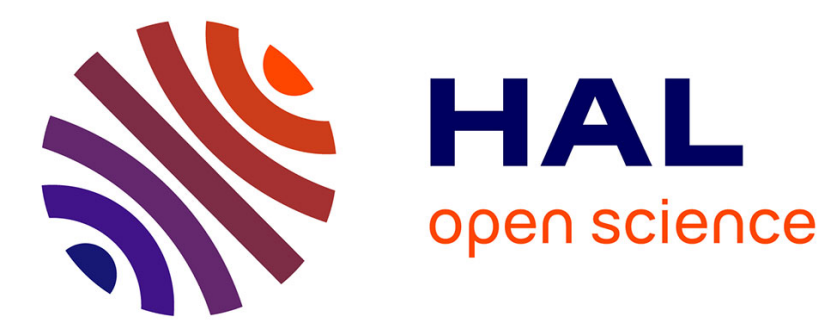

\title{
Virtual Validation of Automotive Measurement Services Based on JT (ISO 14306:2012)
}

\author{
Andreas Faath, Alexander Christ, Reiner Anderl, Frank Braunroth
}

\section{To cite this version:}

Andreas Faath, Alexander Christ, Reiner Anderl, Frank Braunroth. Virtual Validation of Automotive Measurement Services Based on JT (ISO 14306:2012). 12th IFIP International Conference on Product Lifecycle Management (PLM), Oct 2015, Doha, Qatar. pp.505-515, 10.1007/978-3-319-33111-9_46 . hal-01377477

\section{HAL Id: hal-01377477 \\ https://hal.inria.fr/hal-01377477}

Submitted on 7 Oct 2016

HAL is a multi-disciplinary open access archive for the deposit and dissemination of scientific research documents, whether they are published or not. The documents may come from teaching and research institutions in France or abroad, or from public or private research centers.
L'archive ouverte pluridisciplinaire HAL, est destinée au dépôt et à la diffusion de documents scientifiques de niveau recherche, publiés ou non, émanant des établissements d'enseignement et de recherche français ou étrangers, des laboratoires publics ou privés. 


\title{
Virtual Validation of Automotive Measurement Services based on JT (ISO 14306:2012)
}

\author{
Andreas Faath ${ }^{1}$, Alexander Christ ${ }^{1}$, Reiner Anderl ${ }^{1}$, Frank Braunroth ${ }^{2}$, \\ ${ }^{1}$ Technische Universität Darmstadt, Department of Computer Integrated Design, Darmstadt, \\ Germany \\ faath, christ, anderl\}@ dik.tu-darmstadt.de \\ ${ }^{2}$ GME Engineering Measurements \& Calibration Service, Rüsselsheim, Germany \\ frank.braunroth@de.opel.com
}

\begin{abstract}
In this paper a concept for the virtual validation of automotive measurement services based on JT (ISO 14306:2012) is introduced. Each physical measuring component has a digital representation, represented by a 3D JT model. These models contain all necessary information of the measurement process and are the basis for the application of physical sensors. The developed concept enables the consistent usage of JT models and the suitable integration of virtual engineering methods into measuring services, resulting in improved efficiency and interoperability. For the implementation, instead of a CAD system, the viewing software Teamcenter Lifecycle Visualization Mockup is used. This leads to cost savings by the reduction of license fees. The concept is validated based on two measuring projects, containing separate component models and a complete vehicle model.
\end{abstract}

Keywords: Automotive, Digitalization, Interoperability, Measurement Services, Virtual Validation

\section{Introduction}

Increasing cost and time pressure in automotive engineering demands efficient computer-aided methods and tools to fulfill future engineering requirements [1]. A consistent digitalization of products and processes offer huge potential to improve interoperability and ensure a defined quality level in the entire product life cycle. Despite the benefits and manifold application possibilities of virtual methods, automotive measurement services often lack a fully digital integration of underlying processes [2]. Measuring services are characterized by media discontinuities and are based on the knowledge of each individual engineer. 3D models or further exact coordinate values, which are necessary to process measurement results in downstream processes precisely, are not existent. Therefore concepts and processes to enable the integration of virtual methods into measuring processes without increasing effort or cost have to be developed. User-friendly implementation software and neutral data formats are used to enable interoperability within heterogeneous system landscapes and 
to pave the way for global measuring services, which use 3D models as basis for measuring orders, physical sensor application, result presentation, and discussion.

\section{Measurement Services in Automotive Engineering}

Measurement Services in automotive engineering play an important role to ensure product quality and vehicle durability. A variety of systems, information and specific data from different domains like product development, simulation and manufacturing have to be integrated into measurement workflows. The ability to analyze single components in test beds and fully equipped vehicles in road tests on proving grounds with different sensors lead to a high complexity and data diversification. Beside strain gauges, accelerometers and force sensors, wheel force sensors are used for vehicle dynamics and operating load measurements [2]. The measuring process is analyzed using SADT [3], [4]. The process is composed of six main activities. The first activity is the order entry in which restrictions and goals are defined. The second activity is the planning of the measurement. This activity processes the information of the order entry and paves the way for the preparation and the whole measuring process. In the third activity images, components and sensors are used to prepare the physical measuring vehicle. The last three activities represent the execution of the measurement process. After the activity "measuring and disassembling" the fifth activity "evaluation" uses statistical analysis to generate usable results, which are used during the result documentation to generate reports and presentations in activity six. Although already established and serving as key enabler for quality insurance, the seamless integration of measurement services in value adding business processes is missing. Today's automotive measurement services often fall short to offer consistent solutions to address the current and future requirements of engineering processes. The integration of standardized data formats like STEP [5], [6], [7] or JT [8] as neutral data formats is a promising approach to ensure data consistency and structure. These formats offer potential for cost savings by the reduction of license fees, to reduce time to market, and to improve interoperability in heterogeneous system landscapes. For a business strategy, the main objective is to establish one standardized data format for all engineering downstream processes [9], [10].

\section{JT as Neutral Downstream Processes Format}

JT (ISO 14306:2012) [8] is a standardized data format which enables the creation and utilization of performant 3D visualization models. JT is well-established in automotive industry and provides a CAD neutral description. The compressible binary format JT contains different geometry representations. Tessellated representations for rough to fine (Levels of Detail, LOD) are provided as well as exact geometry data as boundary representation (B-Rep) and ultra-lightweight representations (ULP). The ultra-lightweight representation is used to achieve small file sizes with high visualization accuracy. Meta data like product structures and product manufacturing information (PMI) can be integrated, as illustrated in Fig. 1 [8], [11]. 


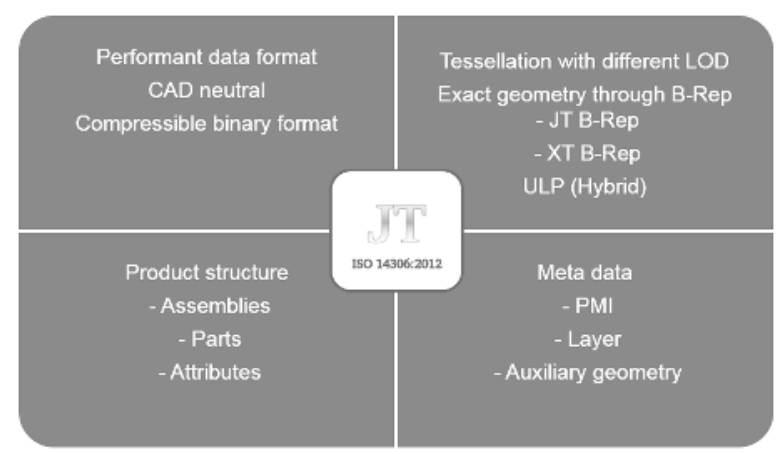

Fig. 1. JT data format [10]

The fields of application for JT are categorized into three groups: 3D visualization, data exchange and collaborative engineering. The different geometry representations integrated in JT enable the efficient and context specific visualization of 3D product data. Viewers in the app technology allow to present JT data on mobile devices. In multi-CAD and design in context applications JT serves, due to the CAD-neutral description as key enabler. 3D product data and meta data can be managed and shared in a suitable form. The integration of accompanying data formats like STEP AP 242 [12] is possible and a preferred approach in automotive industry [13]. Data exchange based on small data volumes and performant $3 \mathrm{D}$ visualization enable the deployment of JT in collaborative engineering. Hence, JT offers huge potential to serve as a standardized, neutral data format in nearly all downstream processes, i.e. simulation, manufacturing, and measurement services [14]. Due to the advantages of JT compared to other data formats and the wide acceptance in science and industry, use cases for the application of JT in downstream processes have been developed by ProSTEP iViP Association and the German automotive industry association (VDA). These use cases provide high level guidelines for the implementation of JT based engineering processes, covering topics from high end visualization and 3D measurement and analysis to supplier integration and manufacturing processes [15]. Nowadays, measurement capabilities in JT are limited to 3D product geometry in a virtual environment. References to physical products cannot be represented. The virtual application of sensors and measurement devices in models is also missing. Especially in course of Industrie 4.0 and the internet of things the consistent linkage of the physical products, its virtual representation and the integration of sensors in cyber-physical systems is becoming prevalent. The integration of JT in automotive measurement services is a first step towards a holistic information integration. The concept is described in the following section. 


\section{Concept for the Virtual Validation of Automotive Measurement Services based on JT}

To provide a basis for the concept, requirements are developed. The main categories are the utilization of neutral data formats and user-friendly software that contain all necessary functions without the condition of extensive CAD knowledge. Furthermore precise coordinate values need to be processed. The final result should be a hundred percent accordance of the 3D-Model, containing the correct sensors at their correct positions and the physical measuring assembly, which serves as a basis of the physical sensor application. Thus the prescribed coordinate values need to be transferred to the test component accurately. In addition, the coordinate values of appliqued sensors must be detectable and adjustable in the 3D model. To ensure the processing and the use of the generated models, measurement results must be visualized structured directly in the 3D model. The concept for the virtual validation of automotive measurement services is divided into four parts. Those are the virtual determination of sensor coordinate values, the virtual positioning of 3D sensor models, the transfer and detection of coordinate values and finally the result presentation on 3D models. Due to the fact that the concept provides the use of available vehicle models, the user is able to mark the desired sensor positions directly in the 3D model. Complex 2D views and markings on these are completely avoided. It is possible to transfer sensor positions by selecting the requested positions qualitatively depending on geometrical requirements. Furthermore prescribed user defined precise coordinate values can be used for sensor positioning. Different layers of visualized coordinate values are used to enable the assignment of corresponding sensor types. Sensor types i.e. accelerators has its own displayed color of the selected coordinate value to recognize the planned sensor type even though all layers are activated. Fig. 2 displays a selected and automatically visualized coordinate value which relates to an accelerator.

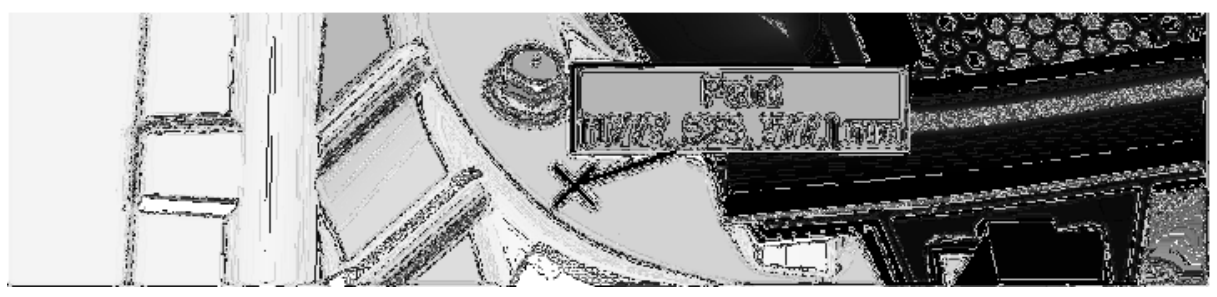

Fig. 2. Coordinate value of selected sensor position

Following the selection and visualization of all desired sensor positions, the associated JT sensor models can be positioned using the integrated coordinate values. The JT sensor models correspond to the physical sensor geometry. This enables the identification of missing mounting space and intersections between sensor models and measuring parts. Possible errors can be fixed by manipulating the sensor positions. To extend the information content of the model, important documents for sensor preparation and application are linked to the sensor models. To avoid incorrect positioning of sensors and resulting effort during the physical sensor application, the created sensor measuring setup is transferred to the user for approval. Fig. 3 shows the 
process of virtual sensor application and approval by the client. As illustrated, the vehicle model is loaded as JT model from the product data management (PDM) system. The sensor models are positioned in their belonging layers and necessary information is linked to the sensor models. If there are no complaints by the user, the next concept component is processed.

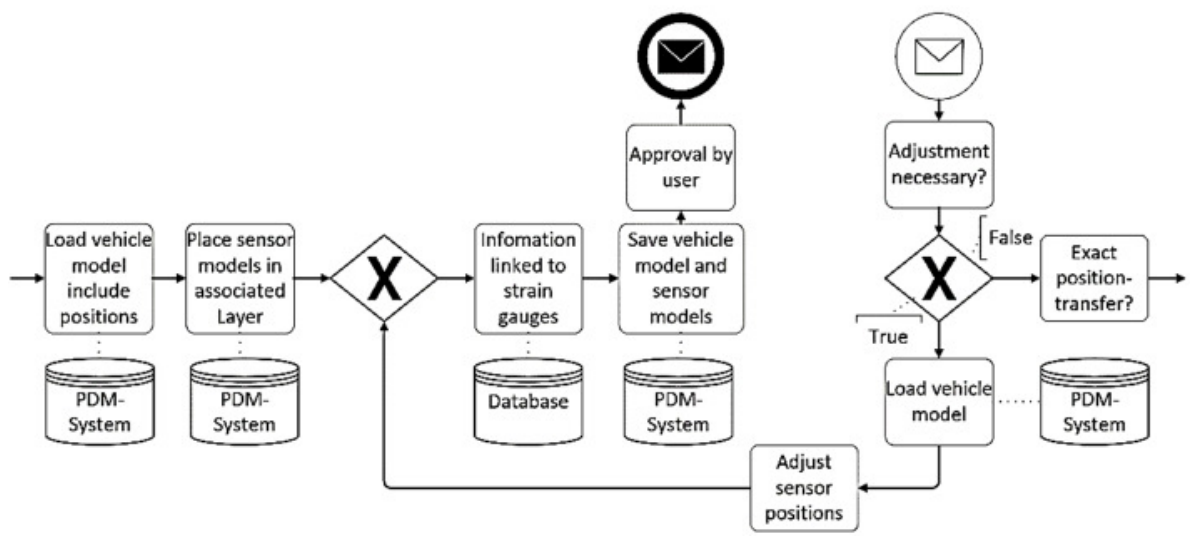

Fig. 3. Process of virtual sensor application an approval

To transfer the coordinate values of the sensor a Coordinate Measuring Machine $(\mathrm{CMM})$ is used. This allows a physical sensor application at their designated positions with high accuracy. The 3D JT models are the basis for the physical attachment and also define the orientation of the sensors. To guarantee an exact correspondence the physical coordinate values are measured and corrected if necessary in the JT models. In the latest development state the process step "measurement and disassembling" is only used for physical components and vehicles. Current analyses focus on the complete virtualization of this process step and the integration into cyber-physical measurement components. For evaluation and documentation JT sensor models and component, respectively vehicle models are used. They function as basis for an efficient visualization of measurement results directly on 3D models, see Fig. 4. The results correspond directly to the measuring positions, including the precise coordinate values.

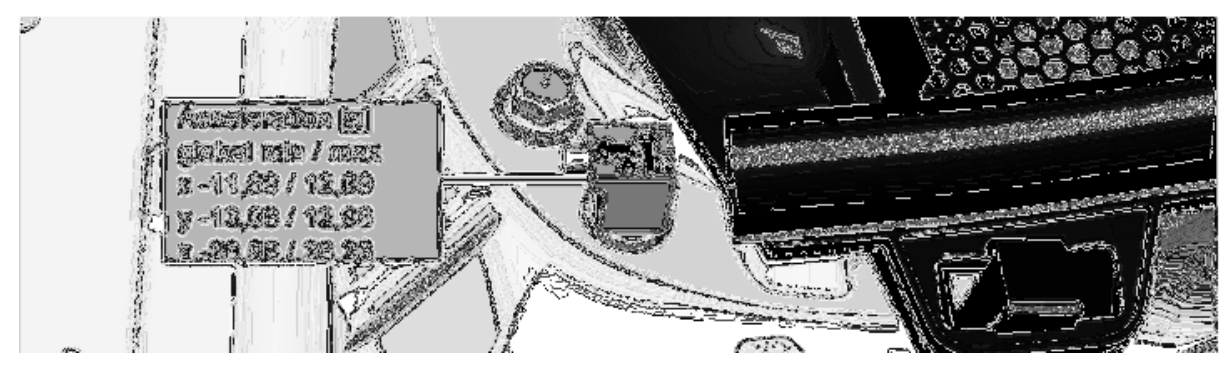

Fig. 4. 3D JT sensor model with measurement results 
Downstream processes, e.g. finite element analysis or multi body simulations are able to process simulation results for iterative optimization processes and to verify simulation results. Furthermore, the use of 3D part models enables the transfer of developed sensor setups to different design levels of the same component, which allows considerable time savings. In addition, the transfer between different design levels provides precise comparability between the different design levels, which enables the valodation of improvement. Due to the virtual validation, the necessary information of the measuring processes is integrated in the 3D model and is independent of the user.

\section{Implementation}

In order to achieve a platform independent implementation of the concept, the usage of CAD software is avoided. This leads to cost savings by the reduction of license fees and prevents the otherwise necessary education of measurement engineers in CAD systems. The 3D JT models serve as basis for the implementation. Meta data such as product structures and assembly information is stored in the XML format. To ensure interoperability in this early development status PLMXML is used, due to the retrieval of 3D component and vehicle models from the Teamcenter PDM system. PLMXML assemblies refer to the assembled JT models. The integration of STEP AP 242 XML in measurement services is intended and part of future research. Additionally, .vf files are used to display Teamcenter Visualization authored sessions and to store snapshots. Since all participants of the measuring process have to work with the 3D models without having CAD experiences and knowledge, the visualization software Teamcenter Visualization Mockup is deployed. As PDM system Siemens Teamcenter is used. To generate the models the $3 \mathrm{D}$ sensor models and vehicle component models have to be modelled before and saved as JT Files. The most important functions of Teamcenter Visualization Mockup are "3D-Measurements", "3D-Selection", “3DLayer Control", "3D-Part Transformation", "3D-Groups", "3D-Markups" and "3DSnapshots". "3D-Measurement" combined with "3D-Selection" allows precise measurement and displaying of qualitatively prescribed sensor positions. In addition, quantitative user defined coordinate values can be selected. "3D-Measurements" are also used to specify distances that are necessary for physical sensor mounting. The "3DSelection" enables the definition of selectable geometric elements. "3D-Layers" are used to structure the displayed coordinate values and sensor models. The function "3DPart Transformation" is used to position the sensor models at their planned positions. Moreover the sensor models are adjusted precisely by customizing their exact position by configured increments. Adjusting the positions of sensor models is required if there are intersections between sensors and vehicle models. New coordinate values can be identified in the "3D Part Transformation" window. Displayed coordinate values have to be corrected. "3D-Groups" are used to group all sensors of the same type or topic to enable a quick and structured visualization of related sensors. Sensors and vehicle components can be grouped to represent special interests of the measurement order. The function "3D-Markups" is important to visualize measurement results or user defined annotations. It is possible to link information, tables or graphs off all kind to the created markups. To prevent overloaded views, the function snapshot is used to save 
predefined views. The function enables the user to save the current view and to include markups, measuring and any other displayed elements. Snapshots are used to represent information of user defined topics, e.g. maximum and minimum results or results during explicit test tracks.

\section{Validation}

The presented concept is validated using two measuring projects. One measuring order processes the vehicle component rear axle, which is prepared using strain gauges. The second measurement order refers to accelerations and several vehicle positions and is prepared by using fourteen accelerometers. Both measuring orders are combined in one project. Therefore, the measurement orders are prepared in separate models and realized in separate processes. Finally, both models which are processed in two independent models, are unified and all components are mounted in one measuring vehicle. As described during the order meeting the user defines sensor positions in coordination with the measurement engineer. The component and vehicle models are the basis for discussions and support decision making in downstream processes. Interoperability is improved since users are able to define sensor positions on their own without having a personal meeting with the measurement engineer. The concept enables the preparation of vehicle models with sensor models without using the physical vehicle component. Remaining time until the prototype components are available can be used to prepare the measurement process. Fig. 5 (left) shows a snapshot of twelve accelerometer positions on a complete vehicle model. On the right side the comparison between JT sensor model and physical sensor is shown. Different sensor type positions are highlighted by an underlying color coding.
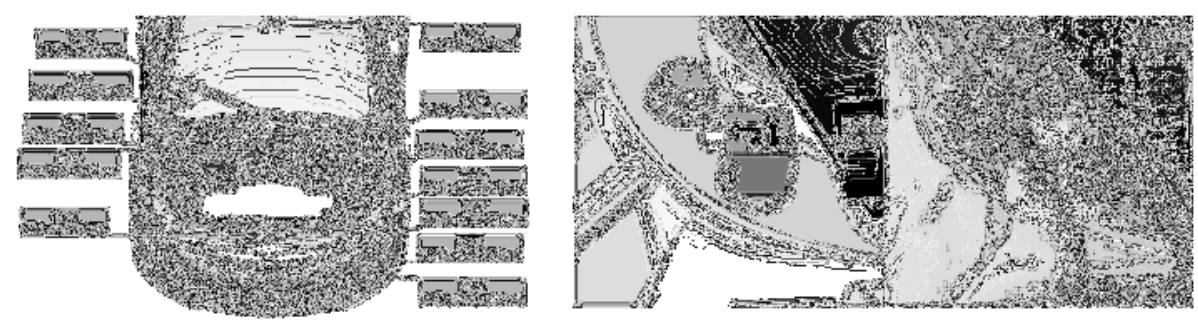

Fig. 5. Defined sensor positions on complete vehicle (left), JT sensor model and physical application (right)

3D measurement machines are used, if an accurate translation of coordinate values to the physical component or vehicle is required. If there is no accurate transformation necessary, the model will work as foundation of qualitative sensor application. In this case it is important to measure the physical sensor positions to receive an exact accordance between component and model. Besides the correct positions, the sensor orientation has to be considered. Therefore information is represented by the utilization of 3D markups. To transfer local sensor accelerations to global vehicle acceleration it is mandatory to retain concurrency between sensor axis and vehicle axis. Marely 
rotations in increments of $90^{\circ}$ are allowed to enable ideal cable routing as shown in Fig. 5 (right). If there is no possibility to orient sensors parallel to vehicle axis, rotation angels can be used to transform the measuring results. Afterwards the model is checked by the user, the physical sensor application is processed, and the sensor positions are detected by the measurement machine. The approval paves the way for a complete faultlessness. The elimination of wrong sensor applications save time and cost. As shown in Fig. 6, the divergences between defined coordinate values and coordinate values of mounted sensors are infinitesimal. Welds are exceptions in case they are not modelled in physical conditions. In this case strain gauges have to be positioned qualitatively next to the weld. A synergistic effect is the control of model quality.

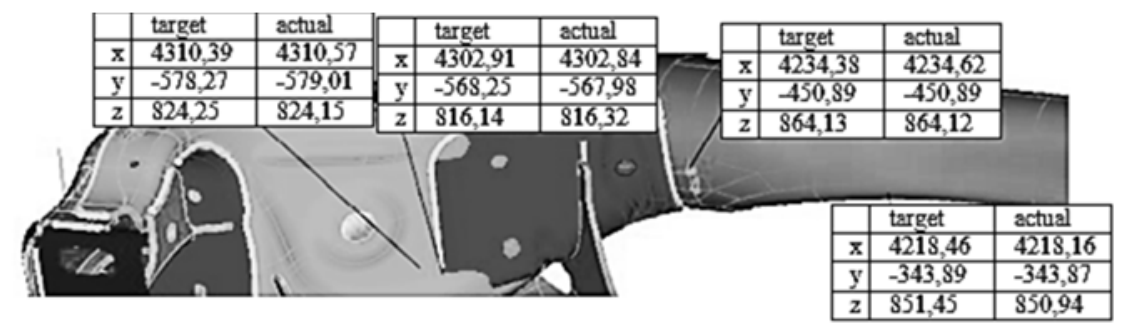

Fig. 6. Results of 3D measurement machine

After the measurement and the evaluation is completed, the most important results and sensor labels are visualized in the model. Fig. 7 shows the mounted rear axis including the sensor labels. The assembly allows to collect several measuring orders in one vehicle model and allows a general survey in which all the measurements of one vehicle can be compared and processed. Different measuring orders are grouped and can be visualized separately if there is no need to display the vehicle context.

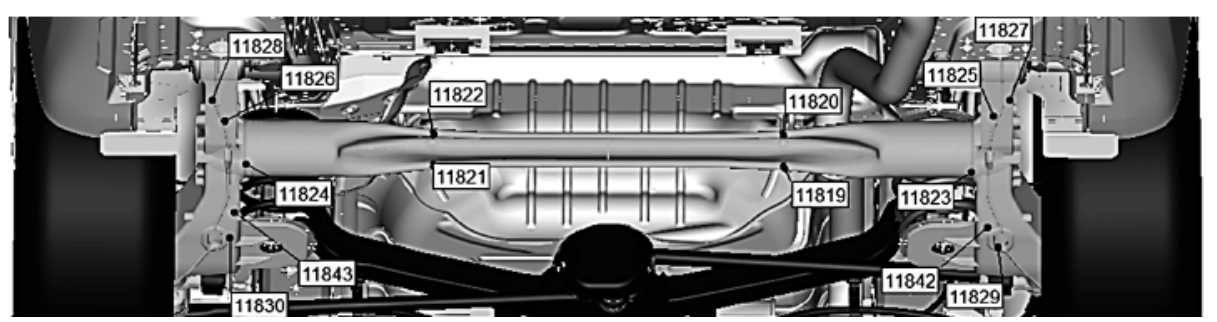

Fig. 7. Mounted rear axis with named strain gauges

Results of different topics are structured in different layers and linked to further information. The final result of the virtual validation of measuring services is a model that contains the vehicle and sensor models as well as their physical positions and measuring results, linked to further information. It delivers an exact comparison between the model and the physical measuring vehicles and is available even after the measuring vehicle is already disassembled. The model is used as collaboration tool, is processed by simulations and allows to display already measured components. This prevent duplication of efforts. Fig. 8 shows the important steps during the validation. 

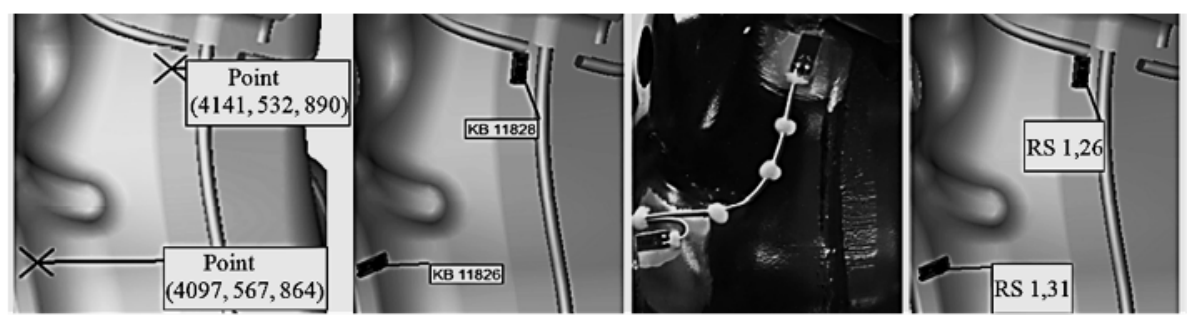

Fig. 8. Main validation steps

\section{Conclusion and Outlook}

In this paper the concept for the virtual validation of measurement services in automotive engineering based on the open standard JT (ISO 14306:2012) was introduced. The concept was implemented in a realistic automotive system landscape and was successfully validated using two measurement projects. Benefits of this approach are the independency of time, place and individual knowledge. The virtual validation of automotive measuring services enables the collaboration based on vehicle models which contain sensor models and all necessary information. This information can be processed several downstream processes. Efficiency and interoperability are increased using lightweight visualization models instead of fully functional CAD models. Consistent processes based on JT prevent unnecessary media discontinuities and duplication of effort, resulting in cost and time savings. In addition, sensor positions and models can be transferred between related components or different design levels of the same component. Knowledge can be aggregated by executing measuring orders and documenting solved problems and best practices in the models. The implementation software TC Vis Mockup offers a manageable functional scope in a user-friendly software environment. Disadvantages are the dependency on already available JT models and the divergence between 3D models and physical components. Table 1 shows the advantages and disadvantages of the virtual validation of measuring services.

Table 1. Advantages and Disadvantages

\begin{tabular}{|l|l|}
\hline Advantages & Disadvantages \\
\hline Usage of precise coordinate values & Dependence on available models \\
\hline Physical application based on models & Absolut positioning of sensor models \\
\hline $\begin{array}{l}\text { Independency of disassembling of } \\
\text { measuring components }\end{array}$ & $\begin{array}{l}\text { Divergence between JT models and } \\
\text { physical components }\end{array}$ \\
\hline Measuring results visualized & \\
\hline Transfer of measuring setups & \\
\hline Knowledge management based on models & \\
\hline User friendly implementation & \\
\hline Improved interoperability & \\
\hline
\end{tabular}


The concept introduced in this paper is a first step for the consistent digitalization of measurement services as well as the integration of the established data format JT in the underlying processes. Future research will focus on the identification and analysis of potential use cases and their specification using the business process model and notation (BPMN). The combination with other standardized data formats like STEPAP 242 is intended. The measurement service analysis was performed mainly for intern GME measurement processes. Further developments will focus on a suitable customer integration to fulfill future business service requirements. To improve interoperability on a global scale, the integration of web services for information retrieval from different IT systems is considered. This could lead to globally distributed automotive measurement services, enabling the utilization of the same vehicle measurement set ups independent of the location. Global users are able to prepare models which function as measurement orders and can be executed and completed through measuring results. Furthermore, the transfer into new industry sectors and the extension to cyber- physical systems is desired [16]. They enable the representation of measuring data on the fly during the road load data acquisition on testing fields. In this case measuring data can be sighted and improved on demand, adding value for all customers of global measurement services.

\section{References}

1. Grimm, K.: Software technology in an automotive company: major challenges. In: Proceedings of the 25th international conference on Software Engineering, pp. 498-503. IEEE Computer Society (2003)

2. Ferry, W. B., Frise, P. R., Andrews, G. T., \& Malik, M. A. (2002). Combining virtual simulation and physical vehicle test data to optimize durability testing. Fatigue \& Fracture of Engineering Materials \& Structures, 25(12), 1127-1134.

3. Ross, D. T., Schoman Jr, K. E.: Structured analysis for requirements definition. Software Engineering, IEEE Transactions on, (1), 6-15 (1977)

4. Marca, D. A., \& McGowan, C.: SADT: structured analysis and design technique. McGraw-Hill (1987)

5. International Organization for Standardization: Industrial automation systems and integration - Product data representation and exchange - Part 203: Application protocol: Configuration controlled 3D design of mechanical parts and assemblies. ISO 10303-203 (2011)

6. International Organization for Standardization: Industrial automation systems and integration - Product data representation and exchange - Part 214: Application protocol: Core data for automotive mechanical design processes. ISO 10303-214 (2010)

7. International Organization for Standardization: Industrial automation systems and integration - JT file format specification for 3D visualization. ISO 14306 (2012)

8. Anderl, R., Trippner, D.: STEP. Standard for the Exchange of Product Model Data. Teubner (2000)

9. Dotzauer, R., Handschuh, S., Fröhlich, A.: Standardized Formats for Visualization - Application and Development of JT. In: 19th ISPE Int. Conf. on Concurrent Engineering, Trier, Germany (2012)

10. Eigner, M., Handschuh, S., Gerhardt, F.: Concept to enrichen lightweight, neutral data formats with CADbased feature technology. In: Computer-Aided Design and Applications, 7(1), pp. 89-99 (2010)

11. Emmer, C., Fröhlich, A., Stjepandic, J.: Advanced Engineering Visualization with Standardized 3D Formats. In: Kovacs, Kochan (eds.): Digital product and process development systems. Springer (2013)

12. International Organization for Standardization: Industrial automation systems and integration - Product data representation and exchange - Part 242: Application protocol: Managed model-based 3D engineering. ISO 10303-242 (2014)

13. ProSTEP iViP Association: JT Content Harmonization. Progress Report and Proposal for JT and Accompanying Formats. Version 3.0 (2013)

14. Christ, A., Anderl, R.: Information Integration using JT Master Models. In: Siemens PLM Connection Americas User Conference, Orlando, FL, USA (2014)

15. ProSTEP iViP Association: JT in der Anwendung. Version 2 (2010)

16. Anderl, R.: Industrie 4.0 - Advanced Engineering of Smart Products and Smart Production. In: Technological Innovations in the Product Development, 19th Int. Seminar on High Technology, Piracicaba, Brazil (2014) 